\title{
How Far Should We Spread Using DS-CDMA in Time and Frequency Selective Fading Channels?
}

\author{
Changqing Zheng \\ Laboratory for Information and Decision Systems, EECS \\ Massachusetts Institute of Technology \\ Cambridge, MA 02139 \\ Email: czheng@mit.edu
}

\author{
Muriel Médard ${ }^{1}$ \\ Laboratory for Information and Decision Systems, EECS \\ Massachusetts Institute of Technology \\ Cambridge, MA 02139 \\ Email: medard@mit.edu
}

\begin{abstract}
Spreading is used to provide diversity in frequency and results in a number of benefits. However, recent literature has shown that for a channel that decorrelates in time and frequency, the capacity of a wideband system in a fading channel goes to zero in the limit of infinite bandwidth (given second and fourth moment constraints that decay with the spreading bandwidth). We first extend to the multiple-access case the upper bound from Médard and Tse [5]. Together with a lower bound based on binary transmission, meaningful bounds to the optimal spreading bandwidth using DS-CDMA are obtained. Given typical system and channel parameters, the minimum spreading bandwidth behaves as a linear function of channel strength. We will show that this result can be meaningfully applied as guidelines in the design of future communication systems.
\end{abstract}

\section{INTRODUCTION}

The information theoretic study of wideband fading channels dates back to the 1960's when Kennedy [2] showed that the capacity of an infinite bandwidth Rayleigh fading channel is equal to that of an infinite bandwidth additive white Gaussian noise (AWGN) channel. In [6], Médard and Gallager showed that very large bandwidths could not be effectively utilized by spread spectrum systems such as DSCDMA that distribute the available power uniformly over both time and frequency. By expressing the input process as an expansion localized in time and frequency of orthonormal set of functions, the fourth moment of each coefficient of this expansion was uniformly constrained. Such a uniform constraint of the fourth moment forced the mutual information to decay to zero inversely with increasing bandwidth. Related to the result of [6] are those of [7], where Subramanian and Hajek showed an alternate derivation using the theory of capacity per unit cost for fourthegy.

Other related results include [8], where Telatar and Tse consider a wideband multipath fading channel and showed that, subject to energy constraint, the capacity of the channel is inversely proportional to the number of resolvable paths. While in this case bandwidth does not directly affect capacity, if the number of paths is very large and the delays between paths are significant, then the number of resolvable paths increases with bandwidth. As the number of resolvable paths increase,

\footnotetext{
${ }^{1}$ This work was supported by the National Science Foundation CAREER grant CCR-00993349 and the MIT-Hewlett-Packard Alliance grant for Advanced Concepts in Wireless Networking
}

capacity suffers. Furthermore, the above result holds even if the receiver is able to perfectly track the timing of the different paths. If the receiver does not have perfect timing knowledge, then the capacity of the channel goes to zero as bandwidth increases to infinity irrespective of the number of resolvable paths.

All of the results above consider the upper limit to capacity and do not offer a range for the optimal spreading bandwidth. Despite the detrimental asymptotic performance of wideband systems, practical experience shows that there may still be an appropriate range within which employing spread spectrum techniques are advantageous. However, none of the existing upper bounds to capacity provide the resolution to study in detail the capacity around practical bandwidths. It is motivated by this desire to study the behavior of DS-CDMA systems in the practical regime that we derive new upper and lower bounds to capacity. In this paper, we will combine an upper bound with a suitable lower bound to capacity to obtain a lower bound for the optimal spreading bandwidth.

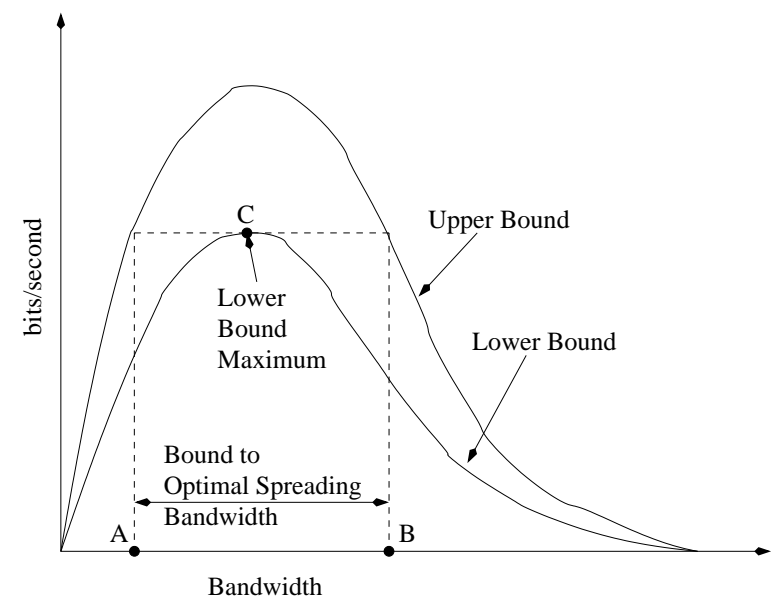

Fig. 1. Bounds to the Optimal Spreading Bandwidth

\section{A. Optimal Spreading Bandwidth}

We now demonstrate how to use an appropriate upper and lower bound to derive a bound for the optimal spreading bandwidth. Suppose that we have a pair of upper and lower 
bounds as in Figure 1. We see that the lower bound achieves the maximum rate at point $C$. Drawing a horizontal line from the maximum of the lower bound, we see that the upper bound achieves this data rate at bandwidths $\mathrm{A}$ and $\mathrm{B}$. If we transmit at a bandwidth below $\mathrm{A}$ or above $\mathrm{B}$, the upper bound tells us the maximum rate we can achieve is less than at point $\mathrm{C}$. In such cases it is more advantageous to use the signaling scheme that achieves the lower bound and transmit at point C. Therefore, points A and B in Figure 1 are bounds to the optimal spreading bandwidth.

We will discuss our channel model in Section II. In Sections III and IV, we find the upper and lower bounds to capacity. We analyze in Section V the capacity bounds in the two-user multiple access scenario for various ranges of parameters and the resulting optimum spreading bandwidth.

\section{Channel Model}

Our channel model is a standard Rayleigh channel that undergoes block fading in time and frequency. Each channel over distinct coherence bandwidths are independent, and transmission occurs over $\mu$ distinct coherence bandwidths. The propagation coefficient for each coherence band remains constant for $T$, the coherence time, after which it changes to a new independent value. These random propagation coefficients are modeled as IID, zero mean, complex circularly symmetric Gaussian random variables. The bandlimited Additive White Gaussian Noise (AWGN) is similarly modeled but normalized to unit variance.

Each coherence band is of size $W$, and samples are obtained at the Nyquist rate of $W$ samples per second. For each received sample in the $i^{t h}$ coherence band at sample time $t, Y[i]_{t}=$ $F_{1}[i]_{t} X_{1}[i]_{t}+F_{2}[i]_{t} X_{2}[i]_{t}+Z[i]_{t}$. Thus within the coherence time $T$ there are a total of $T W$ samples and the received vector in the $i^{t h}$ coherence band over the $j^{t h}$ coherence time is:

$$
\begin{aligned}
\underline{Y}[i]_{j T W+1}^{(j+1) T W}= & F_{1}[i]_{j} \underline{X}_{1}[i]_{j T W+1}^{(j+1) T W}+F_{2}[i]_{j} \underline{X}_{2}[i]_{j T W+1}^{(j+1) T W} \\
& +\underline{Z}[i]_{j T W+1}^{(j+1) T W}
\end{aligned}
$$

There is an average power constraint imposed on the $X_{m}[i]_{t}^{\prime} s$ where a limited amount of power $\mathcal{E}$ is distributed equally to each of the $\mu$ coherence bands, yielding an upper bound on the second moment given by $E\left[X_{m}[i]_{t}^{2}\right] \leq \frac{\mathcal{E}}{\mu}$. Additionally, the peak amplitude is upper bounded by $\left|X_{m}[i]_{t}\right| \leq$ $\frac{\gamma}{\mathcal{E}} \sqrt{E\left[X_{m}[i]_{t}^{2}\right]}$, where $\gamma$ is defined as the peakiness.

\section{UPPER BOUND TO WIDEBAND CAPACITY}

The capacity region of a multiple access channel [1], [3] is the closure of the convex hull of:

$$
\begin{aligned}
C_{1} & \leq I\left(\underline{X}_{1} ; \underline{Y} \mid \underline{X}_{2}\right) \\
C_{2} & \leq I\left(\underline{X}_{2} ; \underline{Y} \underline{X}_{1}\right) \\
C_{1}+C_{2} & \leq I\left(\underline{X}_{1}, \underline{X}_{2} ; \underline{Y}\right)
\end{aligned}
$$

From this result, Lemma 1 gives the capacity region of the upper bound in the two user scenario.
Lemma 1: The upper bound to wideband capacity for the two user scenario based on the channel model described previously in Section II is, for $i \in\{1,2\}$ :

\section{Proof:}

$$
\begin{aligned}
C_{i} \leq & W \mu \log \left(\sigma_{F}^{2} \frac{\mathcal{E}}{\mu}+1\right) \\
& -\frac{\mathcal{E}^{2}}{\gamma^{2} T} \log \left(T W \frac{\gamma}{\sqrt{\mu \mathcal{E}}} \sigma_{F}^{2}+1\right) \\
C_{1}+C_{2} \leq & W \mu \log \left(2 \sigma_{F}^{2} \frac{\mathcal{E}}{\mu}+1\right) \\
& -\frac{\mathcal{E}^{2}}{\gamma^{2} T} \log \left(2 T W \frac{\gamma}{\sqrt{\mu \mathcal{E}}} \sigma_{F}^{2}+1\right)
\end{aligned}
$$

The proof to (2) is as follows, for $i=1$ :

$$
\begin{aligned}
& C_{1}=\lim _{k \rightarrow \infty} \max _{p_{X}} \\
& \left(\frac{1}{k} \sum_{j=1}^{k} \sum_{i=1}^{\mu} \frac{1}{T} I\left(\underline{X}_{1}[i]_{j T W+1}^{(j+1) T W} ; \underline{Y}_{i}[]_{j T W+1}^{(j+1) T W} \mid \underline{X}_{2}[i]_{j T W+1}^{(j+1) T W}\right)\right) \\
& =\lim _{k \rightarrow \infty} \max _{p_{X}} \frac{1}{k} \sum_{j=1}^{k} \sum_{i=1}^{\mu} \frac{1}{T}\left[h\left(\underline{X}_{1}[i]_{j T W+1}^{(j+1) T W} \mid \underline{X}_{2}[i]_{j T W+1}^{(j+1) T W}\right)\right. \\
& \left.-h\left(\underline{X}_{1}[i]_{j T W+1}^{(j+1) T W} \mid \underline{X}_{2}[i]_{j T W+1}^{(j+1) T W}, \underline{Y}[i]_{j T W+1}^{(j+1) T W}\right)\right] \\
& \text { (a) } \\
& \stackrel{(a)}{\leq} \lim _{k \rightarrow \infty} \max _{p_{X}} \\
& \frac{1}{k} \sum_{j=1}^{k} \sum_{i=1}^{\mu} \frac{1}{T}\left[h\left(\underline{X}_{1}[i]_{j T W+1}^{(j+1) T W} \mid F_{2}[i]_{j}, \underline{X}_{2}[i]_{j T W+1}^{(j+1) T W}\right)\right. \\
& \left.-h\left(\underline{X}_{1}[i]_{j T W+1}^{(j+1) T W} \mid F_{2}[i]_{j}, \underline{X}_{2}[i]_{j T W+1}^{(j+1) T W}, \underline{Y}[i]_{j T W+1}^{(j+1) T W}\right)\right] \\
& =\lim _{k \rightarrow \infty} \max _{p_{X}}\left(\frac { 1 } { k } \sum _ { j = 1 } ^ { k } \sum _ { i = 1 } ^ { \mu } \frac { 1 } { T } I \left(\underline{X}_{1}[i]_{j T W+1}^{(j+1) T W} ;\right.\right. \\
& \left.\left.\underline{Y}[i]_{j T W+1}^{(j+1) T W} \mid F_{2}[i]_{j}, \underline{X}_{2}[i]_{j T W+1}^{(j+1) T W}\right)\right) \\
& =\lim _{k \rightarrow \infty} \max _{p_{X}}\left(\frac { 1 } { k } \sum _ { j = 1 } ^ { k } \sum _ { i = 1 } ^ { \mu } \frac { 1 } { T } I \left(\underline{X}_{1}[i]_{j T W+1}^{(j+1) T W} ;\right.\right. \\
& \left.\left.F_{1}[i]_{j} \underline{X}_{1}[i]_{j T W+1}^{(j+1) T W}+\underline{Z}[i]_{j T W+1}^{(j+1) T W}\right)\right) \\
& \stackrel{(b)}{\leq} W \mu \log \left(\sigma_{F}^{2} \frac{\mathcal{E}}{\mu}+1\right)-\frac{\mathcal{E}^{2} \mu}{\gamma^{2} T} \log \left(T W \frac{\gamma^{2}}{\mu \mathcal{E}} \sigma_{F}^{2}+1\right)
\end{aligned}
$$

(a) follows from the properties of conditional entropy.

(b) follows from the same line as that given in the proof of the upper bound to capacity given in [5].

To prove (3), we begin with the definition of capacity as mutual information.

$$
\begin{aligned}
C_{1}+C_{2}= & \lim _{k \rightarrow \infty} \max _{p_{X}}\left(\frac { 1 } { k } \sum _ { j = 1 } ^ { k } \sum _ { i = 1 } ^ { \mu } \frac { 1 } { T } I \left(\underline{X}_{1}[i]_{j T W+1}^{(j+1) T W},\right.\right. \\
& \left.\left.\underline{X}_{2}[i]_{j T W+1}^{(j+1) T W} ; \underline{Y}[i]_{j T W+1}^{(j+1) T W}\right)\right)
\end{aligned}
$$

The fourth central moment of $X_{m}[i]_{t}$ is $\frac{\gamma}{\mu^{2}}$ and its average power constraint is $\frac{\mathcal{E}}{\mu}$. Since there are no sender channel 
side information and all the bandwidth slices are independent, applying the concavity of mutual information in the input distribution results in selecting all the inputs to be IID to maximize the righthand side (RHS) of (4).

First we rewrite mutual information in terms of entropy:

$$
\begin{aligned}
& \frac{1}{T} I\left(\underline{X}_{1}[i]_{j T W+1}^{(j+1) T W}, \underline{X}_{2}[i]_{j T W+1}^{(j+1) T W} ; \underline{Y}[i]_{j T W+1}^{(j+1) T W}\right) \\
= & \frac{1}{T} h\left(\underline{Y}[i]_{j T W+1}^{(j+1) T W}\right)- \\
& \frac{1}{T} h\left(\underline{Y}[i]_{j T W+1}^{(j+1) T W} \mid \underline{X}_{1}[i]_{j T W+1}^{(j+1) T W}, \underline{X}_{2}[i]_{j T W+1}^{(j+1) T W}\right)(5)
\end{aligned}
$$

Now we find a suitable upper bound to (5). First let us upper bound $h(\underline{Y})$

$$
\begin{array}{ll} 
& \frac{1}{T} h\left(\underline{Y}[i]_{j T W+1}^{(j+1) T W}\right) \\
\stackrel{(a)}{\leq} & \frac{1}{T} \log \left((\pi e)^{T W} \mid \Lambda_{\left.\underline{Y}[i]_{j T W+1}^{(j+1) T W} \mid\right)}\right. \\
\stackrel{(b)}{\leq} & \frac{1}{T} \log \left((\pi e)^{T W} \prod_{m=1}^{T W}\left[\sigma_{F}^{2}\left(\sigma_{X_{1, m}}^{2}+\sigma_{X_{2, m}}^{2}\right)+1\right]\right) \\
\stackrel{(c)}{\leq} & W \log (\pi e)+W \log \left(2 \sigma_{F}^{2} \frac{\mathcal{E}}{\mu}+1\right)
\end{array}
$$

(a) follows because entropy is maximized by a Gaussian distribution for a given covariance matrix. Expression (6) gives the entropy of a zero mean complex circularly symmetric Gaussian random vector.

(b) follows from Hadamard's inequality.

(c) follows from the average power constraint.

Next we find a suitable lower bound to $h\left(\underline{Y} \mid \underline{X}_{1}, \underline{X}_{2}\right)$

$$
\begin{array}{ll} 
& \frac{1}{T} h\left(\underline{Y}[i]_{j T W+1}^{(j+1) T W} \mid \underline{X}_{1}[i]_{j T W+1}^{(j+1) T W}, \underline{X}_{2}[i]_{j T W+1}^{(j+1) T W}\right) \\
\stackrel{(a)}{=} & \frac{1}{T} E_{X}\left[\operatorname { l o g } \left((\pi e)^{T W}\right.\right. \\
& \mid \Lambda_{\left.\left.\underline{Y}[i]_{j T W+1}^{(j+1) T W}\left|\underline{X}_{1}[i]_{j T W+1}^{(j+1) T W}, \underline{X}_{2}[i]_{j T W+1}^{(j+1) T W}\right|\right)\right]} \\
\stackrel{(b)}{\geq} & \frac{1}{T} E_{X}\left[\log \left((\pi e)^{T W}\left[\sigma_{F}^{2}\left(\left\|\underline{X}_{1}\right\|^{2}+\left\|\underline{X}_{2}\right\|^{2}\right)+1\right]\right)\right] \\
= & W \log (\pi e)+ \\
& \frac{1}{T} E_{X}\left[\log \left(\sigma_{F}^{2}\left(\left\|\underline{X}_{1}\right\|^{2}+\left\|\underline{X}_{2}\right\|^{2}\right)+1\right)\right]
\end{array}
$$

(a) results from the fact that conditioned on $\underline{X}_{1}[i]_{j T W+1}^{(j+1) T W}$ and $\underline{X}_{2}[i]_{j T W+1}^{(j+1) T W}, \underline{Y}[i]_{j T W+1}^{(j+1) T W}$ is a zero mean, complex circularly symmetric Gaussian random vector.

(b) follows from taking the determinant of the conditional covariance matrix and lower bounding it by:

$$
\left|\Lambda_{\underline{Y} \mid \underline{X}_{1}, \underline{X}_{2}}\right| \geq \sigma_{F}^{2}\left(\left\|\underline{X}_{1}\right\|^{2}+\left\|\underline{X}_{2}\right\|^{2}\right)+1
$$

Owing to the concavity of entropy, we can further restrict our signaling scheme to binary on-off keying where within a coherence time, either all ones or all zeros are transmitted. Therefore, a suitable lower bound to (8) can be obtained by using such a binary signal constellation that satisfies the second moment constraint with equality and subject to the peak amplitude constraint. $X$ is therefore distributed as follows:

$$
X= \begin{cases}0 & \text { with prob. } 1-\frac{\mathcal{E}^{2}}{\gamma^{2}} \\ \frac{\gamma}{\sqrt{\mu \mathcal{E}}} & \text { with prob. } \frac{\mathcal{E}^{2}}{\gamma^{2}}\end{cases}
$$

thus, using this distribution and applying the concavity of the log function, (8) can be lower bounded as:

$$
\begin{aligned}
& \frac{1}{T} h\left(\underline{Y}[i]_{j T W+1}^{(j+1) T W} \mid \underline{X}_{1}[i]_{j T W+1}^{(j+1) T W}, \underline{X}_{2}[i]_{j T W+1}^{(j+1) T W}\right) \\
\geq & W \log (\pi e)+\frac{\mathcal{E}^{2}}{T \gamma^{2}} \log \left(2 T W \frac{\gamma^{2}}{\mu \mathcal{E}} \sigma_{F}^{2}+1\right)
\end{aligned}
$$

Substituting (7) and (9) into (5) and taking the limit in (4) yields the desired result.

Q.E.D

\section{A. Tightness of the Upper Bound}

We know from [5], [6], [7], [8] that in the limit of infinite bandwidth, capacity should converge to zero from above. Therefore, having derived an upper bound in Lemma 1, we now show that (2) and (3) do indeed converge to zero as $\mu \rightarrow \infty$. For (3), the proof is as follows:

$$
\begin{aligned}
& \lim _{\mu \rightarrow \infty} W \mu \log \left(2 \sigma_{F}^{2} \frac{\varepsilon}{\mu}+1\right)-\frac{\varepsilon^{2} \mu}{\gamma^{2} T} \log \left(2 T W \frac{\gamma^{2}}{\mu \varepsilon} \sigma_{F}^{2}+1\right) \\
& \text { let } x=\frac{1}{\mu} \text { and applying L'Hospital's rule: } \\
& =0
\end{aligned}
$$

Therefore, the above derivation shows that (3) does indeed provide a sufficiently tight upper bound to the wideband capacity. The asymptotic tightness of (2) can be shown in a similar manner.

\section{LOWER BOUND TO WIDEBAND CAPACITY}

The upper bound to wideband capacity in the two user case provides little information by itself. Having derived the upper bound, it is now necessary to develop an appropriate lower bound. There are many different possible lower bounds to capacity. We select as our lower bound the achievable rate of a binary signaling scheme with low duty cycle. The transmitter sends binary signals, one of which is located at the origin. As in the upper bound, let each element of $\underline{X}_{m}[i]_{j T W+1}^{(j+1) T W}$ be chosen IID, and $X_{m}[i]_{t} \in\{0, \alpha\}$ with the following distribution:

$$
X_{m}[i]_{t}= \begin{cases}0 & \text { with prob. } 1-p \\ \alpha=\sqrt{\frac{\varepsilon}{\mu p}} \leq \frac{\gamma}{\sqrt{\mu \varepsilon}} & \text { with prob. } p\end{cases}
$$

The above distribution satisfies the second moment constraint with equality and the peak amplitude constraint. Because $\gamma$ does not directly factor into the second moment constraint, varying $\gamma$ in this distribution gives us one extra degree of freedom in adjusting the resulting achievable rate. Since this is a multiple access scenario, the received signal is the sum of all the users's signal, each corrupted by their independent fading coefficient, plus the additive Gaussian noise. 
Once transmitted, the receiver performs MAP detection to recover the transmitted signal. In attempting to decode a particular user's signal, the receiver accounts for the presence of the other user by treating its signal as noise. Lemma 2 gives a lower bound to capacity.

Lemma 2: The lower bound to wideband capacity based on the channel model described in Section II and the particular signaling scheme with MAP detection is:

$$
\begin{aligned}
& C\left(W, \mathcal{E}, \sigma_{F}^{2}, T, \mu, \gamma, p\right) \\
\geq & W \mu\left[(1-r) \log \frac{1}{1-r}+r \log \frac{1}{r}\right] \\
& -W \mu(1-p)\left[\left(1-p_{0}\right) \log \frac{1}{1-p_{0}}+p_{0} \log \frac{1}{p_{0}}\right] \\
& -W \mu p\left[\left(1-p_{\alpha}\right) \log \frac{1}{1-p_{\alpha}}+p_{\alpha} \log \frac{1}{p_{\alpha}}\right] \\
& \text { where } \\
p_{0}= & (1-p) \exp \left(-\beta^{2}\right)+p \exp \left(-\frac{\beta^{2}}{\sigma_{1}^{2}}\right) \\
p_{\alpha}= & (1-p)\left(1-\exp \left(-\frac{\beta^{2}}{\sigma_{1}^{2}}\right)\right)+p\left(1-\exp \left(-\frac{\beta^{2}}{\sigma_{2}^{2}}\right)\right) \\
r= & (1-p) p_{0}+p\left(1-p_{\alpha}\right) \\
\beta^{2}= & \frac{\sigma_{2}^{2}}{\sigma_{2}^{2}-1} \ln \left[\frac{(1-p)^{2} \sigma_{2}^{2}}{p^{2}}\right] \\
\sigma_{1}^{2}= & \frac{\mathcal{E}}{\mu p} \sigma_{F}^{2}+1 \\
\sigma_{2}^{2}= & 2 \frac{\mathcal{E}}{\mu p} \sigma_{F}^{2}+1
\end{aligned}
$$

Proof:

Let $X$ denote the signal transmitted and $Y$ the signal received. During transmission, the signal is corrupted by the multiplicative and additive noise. Applying MAP detection reduces the channel to a discrete binary channel with crossover probabilities $p_{0}=P(Y=\alpha \mid X=0)$ and $p_{\alpha}=P(Y=$ $0 \mid X=\alpha)$.

Let $\mathcal{N}\left(0, \sigma^{2}\right)$ represent the PDF of a zero mean, complex circularly symmetric Gaussian random variable with variance $\sigma^{2}$. For the two user case, the additive noise is the sum of additive receiver noise plus the corruption that results from the presence of the other user's signal. Let $\tilde{Z}[i]_{j}$ be the sum of the contributing additive noise effects, then for a receiver trying to decode user one's signal, $Y[i]_{t}=F_{1}[i]_{t} X_{1}[i]_{t}+\tilde{Z}[i]_{t}$, where $\tilde{Z}[i]_{t}$ is the sum of AWGN and the other user's signal

$$
\begin{aligned}
\tilde{Z}[i]_{t}= & Z[i]_{t}+F_{2}[i]_{t} X_{2}[i]_{t} \\
P_{\tilde{Z}}(\tilde{z})= & (1-p) P_{\tilde{Z}}(\tilde{z} \mid x=0)+p P_{\tilde{Z}}(\tilde{z} \mid x=\alpha) \\
= & (1-p) \mathcal{N}(0,1)+p \mathcal{N}\left(0, \alpha^{2} \sigma_{F}^{2}+1\right) \\
& \text { since } \sigma_{1}^{2}=\alpha^{2} \sigma_{F}^{2}+1 \\
= & (1-p) \mathcal{N}(0,1)+p \mathcal{N}\left(0, \sigma_{1}^{2}\right)
\end{aligned}
$$

since $\sigma_{2}^{2}=2 \alpha^{2} \sigma_{F}^{2}+1$, then we have:

$$
\begin{aligned}
& P_{Y \mid X_{1}}\left(y \mid x_{1}=0\right)=(1-p) \mathcal{N}(0,1)+p \mathcal{N}\left(0, \sigma_{1}^{2}\right) \\
& P_{Y \mid X_{1}}\left(y \mid x_{1}=\alpha\right)=(1-p) \mathcal{N}\left(0, \sigma_{1}^{2}\right)+p \mathcal{N}\left(0, \sigma_{2}^{2}\right)(13)
\end{aligned}
$$

Because of the circular symmetry of the PDF, the detection threshold is determined solely by its magnitude and can be calculated by solving the following equations:

$$
\begin{aligned}
(1-p) P_{Y \mid X_{1}}\left(y \mid x_{1}=0\right) & =p P_{Y \mid X_{1}}\left(y \mid x_{1}=\alpha\right) \\
(1-p)^{2} \frac{1}{\pi} \exp \left(-|y|^{2}\right) & =p^{2} \frac{1}{\pi \sigma_{2}^{2}} \exp \left(-\frac{|y|^{2}}{\sigma_{2}^{2}}\right)
\end{aligned}
$$

solving the above equation and letting $\beta$ be the detection threshold:

$$
|\beta|=|y|=\sqrt{\frac{\sigma_{2}^{2}}{\sigma_{2}^{2}-1} \ln \left[\frac{\sigma_{2}^{2}(1-p)^{2}}{p^{2}}\right]}
$$

Having determined the detection threshold as given in (14), the crossover probabilities can be calculated by integrating over the corresponding decision regions. Since only the magnitude factors into the decision regions, the conditional PDF of the magnitude $\varrho$ of the received signal, by a simple change of variable plus accounting for the phase, is the scaled sum of Rayleigh distributed random variables given by the following:

$$
\begin{aligned}
P_{\varrho \mid X}(\varrho \mid x=0)= & (1-p)(2 \varrho) \exp \left(-\varrho^{2}\right) \\
& +p\left(\frac{2 \varrho}{\sigma_{1}^{2}}\right) \exp \left(-\frac{\varrho^{2}}{\sigma_{1}^{2}}\right) \text { for } \varrho \geq 0 \\
P_{\varrho \mid X}(\varrho \mid x=\alpha)= & (1-p)\left(\frac{2 \varrho}{\sigma_{1}^{2}}\right) \exp \left(-\frac{\varrho^{2}}{\sigma_{1}^{2}}\right) \\
& +p\left(\frac{2 \varrho}{\sigma_{2}^{2}}\right) \exp \left(-\frac{\varrho^{2}}{\sigma_{2}^{2}}\right) \text { for } \varrho \geq 0
\end{aligned}
$$

Using the detection threshold given by (14), the crossover probabilities $p_{0}$ and $p_{\alpha}$ are:

$$
\begin{aligned}
& p_{0}=(1-p) \exp \left(-\beta^{2}\right)+p \exp \left(-\frac{\beta^{2}}{\sigma_{1}^{2}}\right) \\
& p_{\alpha}=(1-p)\left(1-\exp \left(-\frac{\beta^{2}}{\sigma_{1}^{2}}\right)\right)+p\left(1-\exp \left(-\frac{\beta^{2}}{\sigma_{2}^{2}}\right) 6\right)
\end{aligned}
$$

Given the crossover probabilities, the probability $r$ of receiving an $\alpha$ can be easily calculated:

$$
r=P_{Y}(y=\alpha)=(1-p) p_{0}+\left(1-p_{\alpha}\right) p
$$

Let $\mathcal{H}(a)=a \log \frac{1}{a}+(1-a) \log \frac{1}{1-a}$, the mutual information of a single use of the discrete binary channel is given by:

$$
\begin{aligned}
I(Y ; X) & =H(Y)-E_{x}[H(Y \mid X=x)] \\
I(Y ; X) & =\mathcal{H}(r)-p \mathcal{H}\left(p_{\alpha}\right)-(1-p) \mathcal{H}\left(p_{0}\right)
\end{aligned}
$$

Substituting $r, p_{0}, p_{\alpha}$ from (17), (15), and (16), respectively, into (18), and multiplying by $W \mu$ to account for the $W \mu$ uses of the channel per second yields the capacity in bits/second as given in (11).

Q.E.D.

\section{ANALysis of RESUlts}

Because we derived the lower bound by treating the other user's signal as noise, both users achieve the same rate in the lower bound. To make for meaningful comparison, for the rest of the paper, let us compare the upper and lower bounds for the case where $R_{1}=R_{2}$. 


\section{A. Lower Bound to Optimal Spreading Bandwidth}

Having derived suitable upper and lower bounds to capacity, we now try to determine the optimal spreading bandwidth using the method outlined in Section I-A. Figure 2 is a plot, in $\log$-log scale, of the upper and lower bounds using common channel parameters $\left(W=100 \mathrm{KHz}, \sigma_{F}^{2}=16, \gamma=100\right.$, $T=0.1 \mathrm{sec}, \mathcal{E}=20$, and $p=0.04$ ). We see that the maximum rate achievable with the binary signaling scheme is approximately 4.2 Mbits per second. By drawing a horizontal line from the maximum of the lower bound, the upper bound achieves this data rate between spreading bandwidths $\mu=15$ and $\mu=16$ coherence bands, equal to $1.6 \mathrm{Mbits} / \mathrm{sec}$ for the lower bound to the optimal spreading bandwidth. Because the upper bound decays very slowly, the maximum spreading bandwidth is so large that it is not of any practical value.

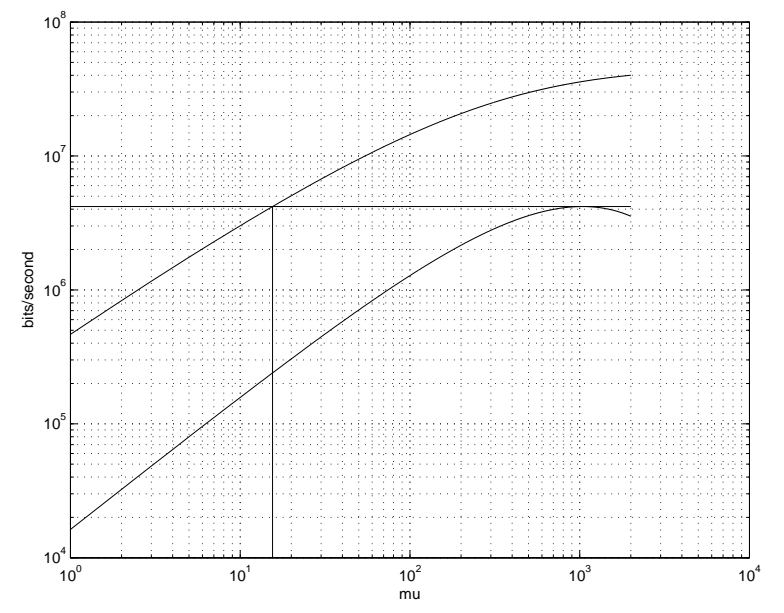

Fig. 2. Multi User Upper and Lower Bound

\section{B. Sensitivity to $\sigma_{F}^{2}$}

The parameter $\sigma_{F}^{2}$ represents the energy of the propagation coefficient, and is particularly significant in the determining the capacity of the lower bound. In the lower bound, because MAP detection is used, $\sigma_{F}^{2}$ determines detection threshold and therefore determines the crossover probabilities. As can be seen from the equation of $\beta$ as a function of $\sigma_{F}^{2}$, a greater $\sigma_{F}^{2}$ yields a lower probability of error. Therefore, the lower bound to capacity would be expected to increase significantly as $\sigma_{F}^{2}$ increases.

To see how the minimum spreading bandwidth varies with $\sigma_{F}^{2}$, we run a number of trials using the same parameters in Section V-A, but varying $\sigma_{F}^{2}$. As the plot of the minimum number of coherence bands $\mu$ to spread to vs. $\sigma_{F}^{2}$ in Figure 3 shows, there is a linear relationship between the energy of the fading coefficient $\sigma_{F}^{2}$ and minimum speading bandwidth $\mu$.

One way to interpret this graph is to view the energy of the propagation coefficient $\sigma_{F}^{2}$ as a metric of the channel power. Since the additive noise power has already been normalized to unity, Figure 3 says that given the user transmits with the above parameters, the minimum number of coherence bands $\mu$ to spread is a linear function of $\sigma_{F}^{2}$. As an example, for $\sigma_{F}^{2}=12$, the minimum number of coherence bands to spread is $\mu=12$. Since each coherence bandwidth is of size $W=100 \mathrm{KHz}$, that means given the system parameters, the system should be spread to $1.2 \mathrm{MHz}$. Considering that the commercial DSCDMA system IS-95 transmits at a bandwidth of $1.25 \mathrm{MHz}$, these results serve as meaningful guidelines for designing future communication systems.

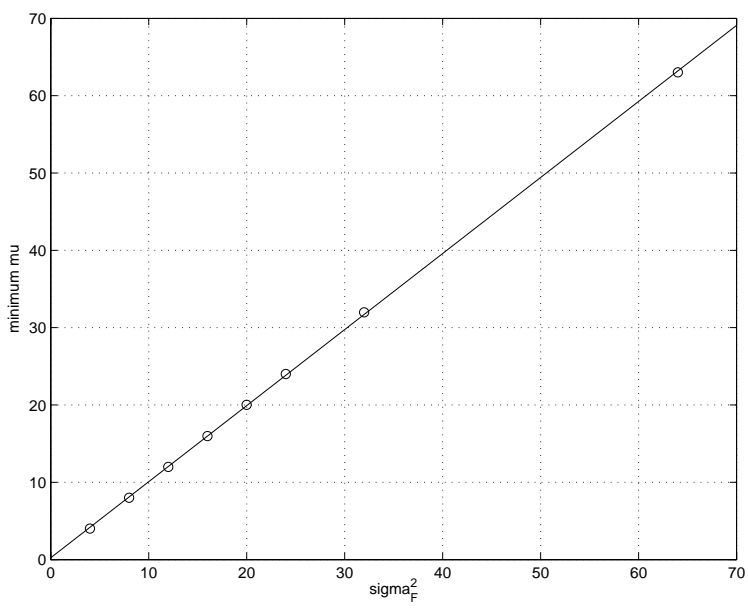

Fig. 3. $\sigma_{F}^{2}$ vs. minimum $\mu$

\section{CONCLUSION}

By deriving a suitable upper and lower bound to capacity, we show in this paper that the minimum speading bandwidth (manifested as $\mu$ ) is a linear function of the energy of the propagation coefficient $\sigma_{F}^{2}$. The example in Section V$\mathrm{B}$ shows that given those channel parameters, the minimum spreading bandwidth is $1.1 \mathrm{MHz}$, which is very close to the $1.25 \mathrm{MHz}$ at which the commercial DS-CDMA system IS-95 transmits. This shows that this result can meaningfully applied as guidelines in the design of future communication systems.

\section{REFERENCES}

[1] R. Ahlswede, "Multi-way Communication Channels," Proceedings of 2nd International Symposium on Information Theory (Tsahkadsor, Armenian S.S.R.), pp. 23-52, Prague, 1971.

[2] R.S. Kennedy, Fading Dispersive Communication Channels, New York: Wiley-Interscience, 1969.

[3] H. Liao, Multiple Access Channels, Ph.D. Thesis, University of Hawaii, 1972.

[4] M. Médard, "Bound on Mutual Information for DS-CDMA Spreading over Independent Fading Channels", proceedings of Asilomar Conference on Signal, Systems and Computers, November 1997, pp. 187-191.

[5] M. Médard, D.N.C. Tse, "Spreading in Block-Fading Channels", Conference Record of the 34th Asilomar Conference on Signal, Systems and Computers, 2000, vol. 2, pp. 1598-1602.

[6] M. Médard, R.G. Gallager, "Bandwidth Scaling for Fading Multipath Channels", IEEE Transactions on Information Theory, Vol. 48, No. 4, April, 2002.

[7] V.G. Subramanian, B. Hajek, "Broad-Band Fading Channels: Signal Burstiness and Capacity," IEEE Transactions on Information Theory, Vol. 48, No. 4, April, 2002.

[8] E. Telatar, D.N.C. Tse, "Capacity and Mutual Information of Wideband Multipath Fading Channels", IEEE Transactions on Information Theory, Vol. 46, No. 4, July 2000. 